\title{
Point Model Equations for Neutron Correlation Counting: Extension of Böhnel's Equations to Any Order
}

\author{
Andrea Favalli ${ }^{\mathrm{a}}$, Stephen Croft ${ }^{\mathrm{b}}$, Peter Santi ${ }^{\mathrm{a}}$ \\ ${ }^{a}$ Safeguards Science \& Technology Group, \\ Non-proliferation and Nuclear Engineering Division \\ Los Alamos National Laboratory, MS E540, Los Alamos, NM 87545, USA. \\ Phone: +1 5056671429 \\ e-mail: afavalli@lanl.gov \\ ${ }^{\mathrm{b}}$ Safeguards \& Security Technology \\ Nuclear Security and Isotope Technology Division \\ Oak Ridge National Laboratory \\ One Bethel Valley Road, PO Box 2008, MS-6166 \\ Oak Ridge, TN 37831-6166, USA.
}

\begin{abstract}
Various methods of autocorrelation neutron analysis may be used to extract information about a measurement item containing spontaneously fissioning material. The two predominant approaches being the time correlation analysis (that make use of a coincidence gate) methods of multiplicity shift register logic and Feynman sampling. The common feature is that the correlated nature of the pulse train can be described by a vector of reduced factorial multiplet rates. We call these singlets, doublets, triplets etc. Within the point reactor model the multiplet rates may be related to the properties of the item, the parameters of the detector, and basic nuclear data constants by a series of coupled algebraic equations - the so called point model equations. Solving, or inverting, the point model equations using experimental calibration model parameters is how assays of unknown items is performed. Currently only the first three multiplets are routinely used. In this work we develop the point model equations to higher order multiplets using the probability generating functions approach combined with the general derivative chain rule, the so called Faà di Bruno Formula. Explicit expression up to $5^{\text {th }}$ order are provided, as well the general iterative formula to calculate any order. This work represents the first necessary step towards determining if higher order multiplets can add value to nondestructive measurement practice for nuclear materials control and accountancy.
\end{abstract}

Key words: point model equations; neutron multiplicity counting; neutron coincidence counting; time correlation analysis

(C) 2015. This manuscript version is made available under the Elsevier user license http://www.elsevier.com/open-access/userlicense/1.0/ 


\section{Introduction}

Passive autocorrelation neutron counting is a commonly used technique for fissile material control and accountancy. The autocorrelation reduced neutron factorial multiplet rates extracted from experimental pulse trains only have meaning for quantitative analysis of special nuclear materials if they can be interpreted in terms of (generally) known properties of the detection system and the physical properties of the item that are of interest, some of which might be known but some of which are usually the subject of the assay. During calibration all properties of a measurement item might be known, and this allows us to determine model parameters to be applied to the assay of items of unknown characteristics. In the current state of the practice, the data interpretation model most widely used is the point-reactor kinetics model which is especially convenient because it leads to expressions in closed analytical form which are straightforward to work with. These equations allow us to take the multiplet rates determined experimentally from the pulse train and invert (or solve) the theoretical point model relationships to obtain estimates of the unknown model parameters which are taken to be meaningful and informative about the corresponding physical properties of the item. In the case of traditional multiplicity counting in which three experimental rates are measured, the point model expressions are well known [1] and the three rates derived from the particular form of multiplicity shift register analysis are referred to as singles, doubles and triples, SDT. These are directly proportionate to the corresponding multiplet rate, differing by factors dependent on the detector and choice of coincidence gating structure. The SDT-expressions may be solved for three unknown parameters analytically. Here we extend the results to higher order (which in the multiplicity shift register example would be quads (Q) and pents (P)) so that the next two higher order measured multiplet rates may also be included in the assay analysis when statistically viable and appropriate to do so. When working with higher order multiplets it is generally easier to perform the necessary inversion by numerical iteration than by analytical methods. The equations are well behaved and so for conforming data (i.e. positive rates with reasonable precision) a simple iterative numerical algorithm is adequate and fast. One can also make use of useful combinations of counting rates which are independent of both the spontaneous fission rate and the detection efficiency parameters.

\section{Theory Development}

Hage and Cifarelli [2] derived the emergent reduced factorial moments from a point-like item in the framework of the prompt-superfission concept [3] using event tree analysis. Böhnel [4], independently arrived at equivalent and general expressions using Probability Generating Function (PGF) theory.

Based on these two foundational works the general expression for the $\mathrm{n}^{\text {th }}$ order detected multiplet rate may be expressed as the sum of contributions from fission cascades initiated by the

spontaneously fissioning source term, $F_{S}$ fissions per sec, and the $(\alpha, \mathrm{n})$ source term, $S_{\alpha},(\alpha, \mathrm{n})$ reactions per sec. We can express this behavior mathematically as follows: 


$$
R_{n}=\left(\varepsilon M_{L}\right)^{n}\left[c_{S n} F_{S}+c_{\alpha n} S_{\alpha}\right]=F_{S}\left(\varepsilon M_{L}\right)^{n} L_{n}
$$

where $\varepsilon$ is the detection efficiency of the external detector in counts per emergent neutron, $M_{L}$ is the leakage self-multiplication, the coefficents $c_{S n}$ and $c_{\alpha n}$ are groupings of the spontaneous and induced fission factorial moments formed over the respective prompt neutron multiplicity distributions, and $L_{n}$ is an $(\mathrm{n}-1)^{\text {th }}$ order polynomial in terms of the parameter $\left(\frac{M_{L}-1}{v_{I 1}-1}\right)$. In this work an expression for $L_{n}$ will be developed and the physical meaning of $\left(\frac{M_{L}-1}{v_{I 1}-1}\right)$ will be made clearer later. In the traditional application of the basic point model the c-coefficients are taken to be known constants insensitive to the nature of the item (e.g. the same as the calibration items).

In the present work we first derive the analytical multiplet expressions using the approach of Böhnel [4] up to fifth order. The results for $R_{1}, R_{2}$, and $R_{3}$ are however already well known [1]. The result for $R_{4}$ is less well known but has been developed previously in the context of the Weighted Point Model (WPM), which is an attempt to compensate for the spatial simplification in the basic point model [5], and our expression agrees with that independent work which just stated the result without proof. The result for $R_{5}$ is entirely new based on our literature search but has been derived independently by the present authors on different occasions as a check. In the second part of the paper, for the first time we will generalize the approach to arrive to the equations for the general order $R_{n}$.

\section{Statement of Results}

For convenience we shall now state the results because it is valuable to see where we are heading as we move through the derivation, and it provide the opportunity to define the notation for the model parameters The expectation values for the reduced factorial multiplets up to $5^{\text {th }}$ order satisfy the following relationships:

$$
\begin{aligned}
& R_{1}=\frac{F_{S}\left(\varepsilon M_{L}\right)}{1}\left[v_{S 1}(1+\alpha)\right] \\
& R_{2}=\frac{F_{S}\left(\varepsilon M_{L}\right)^{2}}{2}\left[v_{S 2}+v_{S 1}(1+\alpha) v_{I 2}\left(\frac{M_{L}-1}{v_{I 1}-1}\right)\right] \\
& R_{3}=\frac{F_{S}\left(\varepsilon M_{L}\right)^{3}}{6}\left[v_{S 3}+\left(v_{S 1}(1+\alpha) v_{I 3}+3 v_{S 2} v_{I 2}\right)\left(\frac{M_{L}-1}{v_{I 1}-1}\right)+3 v_{S 1}(1+\alpha) v_{I 2}^{2}\left(\frac{M_{L}-1}{v_{I 1}-1}\right)^{2}\right] \\
& R_{4}=\frac{F_{S}\left(\varepsilon M_{L}\right)^{4}}{24}\left[v_{S 4}+\left(v_{S 1}(1+\alpha) v_{I 4}+4 v_{S 2} v_{I 3}+6 v_{S 3} v_{I 2}\right)\left(\frac{M_{L}-1}{v_{I 1}-1}\right)+\left(10 v_{S 1}(1+\alpha) v_{I 2} v_{I 3}+\right.\right. \\
& \left.\left.15 v_{S 2} v_{I 2}^{2}\right)\left(\frac{M_{L}-1}{v_{I 1}-1}\right)^{2}+15 v_{S 1}(1+\alpha) v_{I 2}^{3}\left(\frac{M_{L}-1}{v_{I 1}-1}\right)^{3}\right]
\end{aligned}
$$




$$
\begin{aligned}
& R_{5}=\frac{F_{S}\left(\varepsilon M_{L}\right)^{5}}{120}\left[v_{S 5}+\left(v_{S 1}(1+\alpha) v_{I 5}+5 v_{S 2} v_{I 4}+10 v_{S 3} v_{I 3}+10 v_{S 4} v_{I 2}\right)\left(\frac{M_{L}-1}{v_{I 1}-1}\right)+\left(v_{S 1}(1+\right.\right. \\
& \left.\alpha)\left(15 v_{I 2} v_{I 4}+10 v_{I 2}^{3}\right)+60 v_{S 2} v_{I 2} v_{I 3}+45 v_{S 3} v_{I 2}^{2}\right)\left(\frac{M_{L}-1}{v_{I 1}-1}\right)^{2}+\left(105 v_{S 1}(1+\alpha) v_{I 2}^{2} v_{I 3}+\right. \\
& \left.\left.105 v_{S 2} v_{I 2}^{3}\right)\left(\frac{M_{L}-1}{v_{I 1}-1}\right)^{3}+105 v_{S 1}(1+\alpha) v_{I 2}^{4}\left(\frac{M_{L}-1}{v_{I 1}-1}\right)^{4}\right]
\end{aligned}
$$

In these expressions the following definitions apply.

$F_{S}=m_{e f f} g$ is the spontaneous fission rate of the source nuclide taking place inside the item, fissions per sec. It is the product of the effective mass of the reference spontaneously fissioning source nuclide, $m_{e f f}$, in grams and its specific spontaneous fission rate, $g$ in fissions per sec per gram.

$\varepsilon$ is the neutron detection efficiency, singles counts per neutron emerging from the item.

$\alpha$ is the ratio of the (random in time neutron)-to-(SF,n) production where in the present context the random in time production includes $(\alpha, n)$-reactions and delayed-neutrons following fission. It is common practice, however, to ignore delayed neutron contribution in comparison to the much larger $(\alpha, n)$ production rate in special nuclear material oxides. Thus we may write to a good approximation $\alpha \approx S_{\alpha} / F_{S} v_{S 1}$.

$v_{\mathrm{Si}}$ is the $\mathrm{i}^{\text {th }}$ factorial moment of the normalized SF prompt neutron multiplicity emission distribution, $\mathrm{P}_{\mathrm{S}}(v)$. That is to say, $v_{\mathrm{S} 1}=\sum_{v=1}^{\max } v \cdot \mathrm{P}_{\mathrm{S}}(v), v_{\mathrm{S} 2}=\sum_{v=2}^{\max } v \cdot(v-1)$. $\mathrm{P}_{\mathrm{S}}(v)$, etc. In general $v_{S i}=\sum_{v=\mathrm{i}}^{\max }\left(\begin{array}{l}v \\ i\end{array}\right) \cdot \mathrm{P}_{\mathrm{S}}(v)$, where max is the highest value of $v$ observed. Division by $i$ ! converts the factorial moments into reduced factorial moments and our point model expressions as listed incorporate these factors explicitly in the leading multiplier. Similarly, $v_{\mathrm{Ii}}$ is the $\mathrm{i}^{\text {th }}$ factorial moment of the normalized Induced Fission (IF) prompt neutron multiplicity emission distribution, $\mathrm{P}_{\mathrm{I}}(v)$.

$M_{L}$ is the leakage self-multiplication factor, which within the framework of the in the pointreactor kinetics model is related to the neutron probability of fission, $p_{f}$, the neutron probability of parasitic capture inside the item, $p_{c}$, and the total self-multiplication, $M_{T}$, by the following relations [3]:

$$
\begin{aligned}
& M_{T}=\frac{1}{1-p_{f} v_{I 1}} \\
& M_{L}=\left(1-p_{f}-p_{c}\right) M_{T}
\end{aligned}
$$

The factor $\left(1-p_{f}-p_{c}\right)=p_{L}$ we recognize as the leakage or escape probability.

$p_{f} \cdot M_{T}=\frac{M_{T}-1}{v_{I 1}}=\frac{M_{L}-1}{v_{I 1}-1-p_{c} / p_{f}} \approx \frac{M_{L}-1}{v_{I 1}-1}$, if $p_{c} / p_{f} \ll 1$ 
From which we see that, in the limit that the probability of capture to fission inside the item is small, we can approximate $p_{f} . M_{T}$ by $\left(\frac{M_{L}-1}{v_{I 1}-1}\right)$, and this is what has been done in the point model expressions listed above. The reason for doing this is so as to limit the number of unknown model parameters that need to be solved for from the limited experimental data (number of multiplet rates) available. We note that $p_{f} . M_{T}$ has a special physical interpretation. It is the number of induced fissions per source neutron, and we may exploit this fact to partition the point model equations in intuitive ways. Following common practice the results are normally written exclusively in terms of $M_{L}$ however, because we have an underdetermined problem and wish to reduce the number of unknown model parameters carried.

The derivation of the expressions for pentuplets (or quintuplets) is a requirement to correct the quads $\left(4^{\text {th }}\right.$ order) for rate losses in some algebraic dead time correction formalisms such as Hage and Cifarelli [6], Baeten et al. [7], Hauck et al.[8], and Croft and Favalli $[9,10]$. Quads provide the way to solve for 4 unknowns $\left(\varepsilon, M_{L} \alpha, m_{e f f}\right)$.

Using the familiar shift register logic, the SDTQP rates are directly proportional to the corresponding multiplet rates. So, for example, the pents rate maybe expressed as $P=f_{p} R_{5}$, where $f_{p}$ is the event triggered gate utilization factor which accounts for the fact that in practice a finite pre-delay and gate width are used. While not the focus of this paper it should be noted that in practice, for the measurement of neutronically isolated objects, the gate utilization factors are treated as characteristics of the detector system and may be determined experimentally by either extrapolation to infinite gate or by counting a ${ }^{252} \mathrm{Cf}$ standard $[11,12]$.

\section{Derivation}

Böhnel [4] sets out in a clear manner how to formulate the PGF for the point model. In particular he derives a general expression for the factorial moments of the emergent multiplicity distribution from a point-like multiplying item with a combined source of spontaneous fission, $\mathrm{SF}$, and $(\alpha, \mathrm{n})$ neutrons inside. A few intermediate results are presented below in order to outline the key steps in applying Böhnel's to obtain the reduced factorial moments present on the pulse train.

In the single energy point model all neutrons are equivalent and so we can treat $(\alpha, n)$ reactions as a special case of SF with $v=1$ in defining the combined source rate. Explicitly, let us define the source of SF by a SF rate $F_{S}$ and an average prompt neutron multiplicity distribution $f_{S}(v)$ for the probability that the number of prompt neutrons liberated following fission will be $v$ where $v$ is a finite positive integer including zero. The $(\alpha, \mathrm{n})$ source is defined by the rate $S_{\alpha}$ and the associated multiplicity distribution $f_{\alpha}=\delta_{v 1}$ where $\delta_{v 1}$ is the Kronecker delta function. The equivalent combined source term is therefore defined as follows:

$F_{C}=F_{S}+S_{\alpha}$ 
$f_{C}(v)=\frac{F_{S} f_{S}+S_{\alpha} f_{\alpha}}{F_{S}+S_{\alpha}}=\frac{F_{S}}{F_{C}}\left(f_{S}(v)+\alpha v_{S 1} \delta_{v 1}\right)$

where we have used the previous relation $\alpha=S_{\alpha} /\left(F_{S} v_{S 1}\right)$ to express $f_{C}(v)$ in a compact form.

The combined source multiplicity distribution $f_{C}(v)$ is normalized to unity on $v \geq 0$ as it should, being a true probability, and we see that the factorial moments derived from it behave as follows:

$F_{C} v_{C 1}=F_{S} v_{S 1}(1+\alpha)$

$F_{C} v_{C n}=F_{S} v_{S n}, n \geq 2$

The PGF describing the emergence of all neutrons (resulting from the prompt (instantaneous)superfission burst formed over all generations) from the point item, and therefore available for detection by an external detector, when only a single neutron was present inside the item initially is given by:

$h(u)=\left(1-p_{f}-p_{c}\right) u+p_{f} f_{I}(h(u))$

where $\left(1-p_{f}-p_{c}\right)=p_{L}$ is the neutron leakage probability, $f_{I}(h)$ is the PGF for the factorial moments of the induced fission prompt neutron multiplicity distribution, and $h(u)$ is a function of $u$. By definition we have:

$\left.\frac{d^{n} f_{I}}{d h^{n}}\right|_{u=1}=v_{I n}, n \geq 1$

To proceed we need also to find expressions for $\left.\frac{d^{n} h}{d u^{n}}\right|_{u=1}$. By successive differentiation we obtain:

$\frac{d h}{d u}=\left(1-p_{f}-p_{c}\right)+p_{f} \frac{d f_{I}}{d h} \frac{d h}{d u}$

Upon rearranging and substituting we obtain:

$\left.\frac{d h}{d u}\right|_{u=1}=\frac{\left(1-p_{f}-p_{c}\right)}{1-p_{f} v_{I 1}}=M_{L}$

Proceeding further:

$\frac{d^{2} h}{d u^{2}}=p_{f}\left[\frac{d^{2} f_{I}}{d h^{2}}\left(\frac{d h}{d u}\right)^{2}+\frac{d f_{I}}{d h} \frac{d^{2} h}{d u^{2}}\right]$

And evaluating at $\mathrm{u}=1$, we get:

$\left.\frac{d^{2} h}{d u^{2}}\right|_{u=1}=M_{L}^{2} \widehat{M}_{T} v_{I 2}$

where as a convenient shorthand we have introduced the notation: 
$\widehat{M}_{T}=\frac{p_{f}}{1-p_{f} v_{S 1}}=p_{f} M_{T} \approx \frac{M_{L}-1}{v_{I 1}-1}$

and, as discussed earlier, the approximate form appearing on the far right hand side of the expression for $\widehat{M}_{T}$ results when $p_{c} / p_{f} \ll 1$ and has long been used in the point model equations to reduce the number of unknowns which have to be solved for.

Continuing in the same fashion:

$\frac{d^{3} h}{d u^{3}}=p_{f}\left[\frac{d^{3} f_{I}}{d h^{3}}\left(\frac{d h}{d u}\right)^{3}+3 \frac{d^{2} f_{I}}{d h^{2}}\left(\frac{d h}{d u}\right) \frac{d^{2} h}{d u^{2}}+\frac{d f_{I}}{d h} \frac{d^{3} h}{d u^{3}}\right]$

$\left.\frac{d^{3} h}{d u^{3}}\right|_{u=1}=M_{L}^{3} \widehat{M}_{T}\left[v_{I 3}+3 v_{I 2}^{2} \widehat{M}_{T}\right]$

$\frac{d^{4} h}{d u^{4}}=p_{f}\left[\frac{d^{4} f_{I}}{d h^{4}}\left(\frac{d h}{d u}\right)^{4}+6 \frac{d^{3} f_{I}}{d h^{3}}\left(\frac{d h}{d u}\right)^{2} \frac{d^{2} h}{d u^{2}}+3 \frac{d^{2} f_{I}}{d h^{2}}\left(\frac{d^{2} h}{d u^{2}}\right)^{2}+4 \frac{d^{2} f_{I}}{d h^{2}}\left(\frac{d h}{d u}\right) \frac{d^{3} h}{d u^{3}}+\frac{d f_{I}}{d h} \frac{d^{4} h}{d u^{4}}\right]$

$\left.\frac{d^{4} h}{d u^{4}}\right|_{u=1}=M_{L}^{4} \widehat{M}_{T}\left[v_{I 4}+10 v_{I 2} v_{I 3} \widehat{M}_{T}+15 v_{I 2}^{3} \widehat{M}_{T}^{2}\right]$

$\frac{d^{5} h}{d u^{5}}=p_{f}\left[\frac{d^{5} f_{I}}{d h^{5}}\left(\frac{d h}{d u}\right)^{5}+10 \frac{d^{4} f_{I}}{d h^{4}}\left(\frac{d h}{d u}\right)^{3} \frac{d^{2} h}{d u^{2}}+15 \frac{d^{3} f_{I}}{d h^{3}}\left(\frac{d h}{d u}\right)\left(\frac{d^{2} h}{d u^{2}}\right)^{2}+10 \frac{d^{3} f_{I}}{d h^{3}}\left(\frac{d h}{d u}\right)^{2} \frac{d^{3} h}{d u^{3}}+\right.$

$\left.5 \frac{d^{2} f_{I}}{d h^{2}}\left(\frac{d h}{d u}\right) \frac{d^{4} h}{d u^{4}}+10 \frac{d^{2} f_{I}}{d h^{2}} \frac{d^{2} h}{d u^{2}} \frac{d^{3} h}{d u^{3}}+\frac{d f_{I}}{d h} \frac{d^{5} h}{d u^{5}}\right]$

$\left.\frac{d^{5} h}{d u^{5}}\right|_{u=1}=M_{L}^{5} \widehat{M}_{T}\left[v_{I 5}+\left(15 v_{I 2} v_{I 4}+10 v_{I 3}^{2}\right) \widehat{M}_{T}+105 v_{I 2}^{2} v_{I 3} \widehat{M}_{T}^{2}+105 v_{I 2}^{4} \widehat{M}_{T}^{3}\right]$

We note that the expressions for $\left.\frac{d^{n} H}{d u^{n}}\right|_{u=1}$ are $(n-1)^{t h}$ order polynomials in $\widehat{M}_{T}$ with a premultiplier of $M_{L}^{n}$.

The neutrons initiating fission chains inside the item do not, of course, always arise singly. Therefore, we must fold the results above, which apply when only a single neutron is present initially, with the full multiplicity distribution of the combined source term. The factorial moment generating function for the emergent neutrons initiated by the multiplicity distribution of the combined source is $H=f_{C}(h(u))$ where $h=h(u)$ is a function of the variable $u$. Successive differentiation using the chain rule gives results similar to those seen before when we successively differentiated the PGF $h(u)$. We obtain:

$$
\begin{aligned}
& \frac{d H}{d u}=\frac{d f_{C}}{d h}\left(\frac{d h}{d u}\right) \\
& \frac{d^{2} H}{d u^{2}}=\frac{d^{2} f_{C}}{d h^{2}}\left(\frac{d h}{d u}\right)^{2}+\frac{d f_{C}}{d h} \frac{d^{2} h}{d u^{2}} \\
& \frac{d^{3} H}{d u^{3}}=\frac{d^{3} f_{C}}{d h^{3}}\left(\frac{d h}{d u}\right)^{3}+3 \frac{d^{2} f_{C}}{d h^{2}}\left(\frac{d h}{d u}\right) \frac{d^{2} h}{d u^{2}}+\frac{d f_{C}}{d h} \frac{d^{3} h}{d u^{3}}
\end{aligned}
$$


$\frac{d^{4} H}{d u^{4}}=\frac{d^{4} f_{C}}{d h^{4}}\left(\frac{d h}{d u}\right)^{4}+6 \frac{d^{3} f_{C}}{d h^{3}}\left(\frac{d h}{d u}\right)^{2} \frac{d^{2} h}{d u^{2}}+3 \frac{d^{2} f_{C}}{d h^{2}}\left(\frac{d^{2} h}{d u^{2}}\right)^{2}+4 \frac{d^{2} f_{C}}{d h^{2}}\left(\frac{d h}{d u}\right) \frac{d^{3} h}{d u^{3}}+\frac{d f_{C}}{d h} \frac{d^{4} h}{d u^{4}}$

$\frac{d^{5} H}{d u^{5}}=\frac{d^{5} f_{C}}{d h^{5}}\left(\frac{d h}{d u}\right)^{5}+10 \frac{d^{4} f_{C}}{d h^{4}}\left(\frac{d h}{d u}\right)^{3} \frac{d^{2} h}{d u^{2}}+15 \frac{d^{3} f_{C}}{d h^{3}}\left(\frac{d h}{d u}\right)\left(\frac{d^{2} h}{d u^{2}}\right)^{2}+10 \frac{d^{3} f_{C}}{d h^{3}}\left(\frac{d h}{d u}\right)^{2} \frac{d^{3} h}{d u^{3}}+$

$5 \frac{d^{2} f_{C}}{d h^{2}}\left(\frac{d h}{d u}\right) \frac{d^{4} h}{d u^{4}}+10 \frac{d^{2} f_{C}}{d h^{2}} \frac{d^{2} h}{d u^{2}} \frac{d^{3} h}{d u^{3}}+\frac{d f_{C}}{d h} \frac{d^{5} h}{d u^{5}}$

The reduced factorial multiplet rates present on the detected pulse train are now obtained by dividing by $n$ ! and imposing the influence of a detector with finite efficiency $\varepsilon$ (the combined action of which is like applying multiplicity shift register time correlation analysis logic with perfect gating, zero pre-delay and infinite coincidence gate width). Thus:

$R_{n}=\varepsilon^{n} \frac{1}{n !} F_{C} v_{C n}=\left.\varepsilon^{n} \frac{1}{n !} F_{C} \frac{d^{n} H}{d u^{n}}\right|_{u=1}$

The algebraic evaluation of this expression for $R_{n}$ is accomplished by substituting term by term for each factor in the expressions for the various $\frac{d^{n} H}{d u^{n}}$ recalling that in these expressions $f=f_{C}$ is the multiplicity distribution of the combined source term so that $\frac{d^{n} f}{d h^{n}} \equiv \frac{d^{n} f_{C}}{d h^{n}}=v_{C n}$, where, as explained earlier, $v_{C 1}=\frac{F_{S}}{F_{C}} v_{S 1}(1+\alpha)$ and $v_{C n}=\frac{F_{S}}{F_{C}} v_{S n}$ for $n \geq 2$, and remembering that we also already have expressions for $\frac{d^{n} h}{d u^{n}}$ in terms of the induced factorial moments. The results for $R_{1}$ through $R_{5}$ were quoted earlier and also justify our initial assertion that one can write $R_{n}=F_{S}\left(\varepsilon M_{L}\right)^{n} L_{n}$ where $L_{n}$ is an $(\mathrm{n}-1)^{\text {th }}$ order polynomial in terms of the parameter $\left(\frac{M_{L}-1}{v_{I 1}-1}\right)$. Given the strict mathematical rules one follows in applying the PGF method closed form parametric expressions of a general nature for the coefficients of $L_{n}$ can be developed (see later). It is also interesting to note that the factorial moments of both the SF and associated IF prompt neutron multiplicity distributions vanish above some (reasonably small) finite order (e.g. typically 8 to 10 depending on the experimental dataset being used). Thus, as the probability of fission $p_{f}$ increases to a value of $1 / v_{I 1}$, the condition for prompt critical, the values of $v_{C n}$ continue to grow but only through the ever deeper folding of the finite number of SF and IF factorial moments as the self-multiplication of the item increases. 


\section{Derivation of the General Order Multiplet Relationship}

We have seen that a multiplet of order $m$ can be written as:

$$
R_{m}=\varepsilon^{m} \frac{F_{C}}{m !} \frac{d^{m}}{d u^{m}} f_{c}(h(u))
$$

By applying Faa' di Bruno's formula $[11,12]$ for the $m^{\text {th }}$ derivative of a composite function, we obtain the following formula:

$$
R_{m}=\varepsilon^{m} \frac{F_{C}}{m !} \sum \frac{m !}{b_{1} ! b_{2} ! \ldots b_{m} !} f_{C}^{(k)}(h(u))\left(\frac{h^{\prime}}{1 !}\right)^{b_{1}}\left(\frac{h^{\prime \prime}}{2 !}\right)^{b_{2}} \ldots\left(\frac{h^{(m)}}{m !}\right)^{b_{m}}
$$

Where the sum is over all the different solutions in nonnegative integers $b_{1}, b_{2} \ldots, b_{m}$ of $b_{1}+$ $2 b_{2}+\cdots+m b_{m}=m$ and $k=b_{1}+\cdots+b_{m}$.

By substitution of $f_{C}^{(k)}(h(u))=v_{C k}$ and $v_{C 1}=\frac{F_{S}}{F_{C}} v_{S 1}(1+\alpha)$ and $v_{C k}=\frac{F_{S}}{F_{C}} v_{S k}$ for $n \geq 2$ (we don't reduce by $\mathrm{m}$ ! for the moment to keep it out of the summation too), and recalling that $h^{\prime}=M_{L}$, the equation becomes:

$$
R_{m}=\varepsilon^{m} F_{S} \frac{1}{m !} \sum \frac{m !}{b_{1} ! b_{2} ! \ldots b_{m} !} v_{S k}\left(\frac{M_{L}}{1 !}\right)^{b_{1}}\left(\frac{h^{\prime \prime}}{2 !}\right)^{b_{2}} \ldots\left(\frac{h^{(m)}}{m !}\right)^{b_{m}}
$$

By observing that $\mathrm{k}=1$ only for $\mathrm{b}_{\mathrm{m}}=1$ and $\mathrm{b}_{1}=0$ per each $1 \neq \mathrm{m}$ (otherwise $\mathrm{b}_{\mathrm{m}}=0$ ), and taking into account the definition of $v_{C 1}$ finally we arrive at the general equation for multiplet of order $\mathrm{m}$ :

$$
R_{m}=\varepsilon^{m} F_{S} \frac{1}{m !}\left(v_{S 1}(1+\alpha) h^{(m)}+\sum \frac{m !}{b_{1} ! b_{2} ! \ldots b_{m-1} !} v_{S k}\left(\frac{M_{L}}{1 !}\right)^{b_{1}}\left(\frac{h^{\prime \prime}}{2 !}\right)^{b_{2}} \ldots\left(\frac{h^{(m-1)}}{(m-1) !}\right)^{b_{m-1}}\right)
$$

Where the sum is over all the different solutions in nonnegative integers $b_{1}, b_{2} \ldots, b_{\mathrm{m}-1}$ of $b_{1}+$ $2 b_{2}+\cdots+(m-1) b_{m-1}=m$ and $k=b_{1}+\cdots+b_{m-1}$.

Eq.36 gives the general expression for multiplet of order $m$, knowing the $m$ derivative of the $h$ function. The next step is to address a general equation to calculate $\frac{d^{n} h}{d u^{n}}$, that for the case of $\mathrm{n}=1$, is equal to $M_{L}$, while for $n>1$, the following equation needs to be solved:

$$
h^{(m)}=p_{f} \frac{d^{n} f_{I}(h(u))}{d u^{n}}
$$


This can be tackled as before, by means of the Faa' di Bruno formula, and the following equation is obtained:

$$
h^{(m)}=p_{f} \frac{d^{n} f_{I}(h(u))}{d u^{n}}=p_{f} \sum \frac{m !}{b_{1} ! b_{2} ! \ldots b_{m} !} f_{c}^{(k)}(h(u))\left(\frac{h^{\prime}}{1 !}\right)^{b_{1}}\left(\frac{h^{\prime \prime}}{2 !}\right)^{b_{2}} \ldots\left(\frac{h^{(m)}}{m !}\right)^{b_{m}}
$$

Where the sum is over all the different solutions in nonnegative integers $b_{1}, b_{2} \ldots, b_{m}$ of $b_{1}+$ $2 b_{2}+\cdots+m b_{m}=m$ and $k=b_{1}+\cdots+b_{m}$.

By observing that $k=1$ only for $b_{m}=1$ and $b_{1}=0$ for each 1 such as $1 \neq m$ (otherwise $b_{m}=0$ ), and remembering $\left.\frac{d^{n} f_{I}}{d h^{n}}\right|_{u=1}=v_{I n}$, and collecting from right side $h^{(m)}$, eq.38 becomes:

$h^{(m)}=\frac{p_{f}}{\left(1-p_{f} v_{I 1}\right)} \sum \frac{m !}{b_{1} ! b_{2} ! \ldots b_{m-1} !} v_{I k}\left(\frac{h^{\prime}}{1 !}\right)^{b_{1}}\left(\frac{h^{\prime \prime}}{2 !}\right)^{b_{2}} \ldots\left(\frac{h^{(m-1)}}{(m-1) !}\right)^{b_{m-1}}$

And finally, recalling the definitions given previously, the final equation for $h^{(m)}$ is obtained:

$$
h^{(m)}=\left(\frac{M_{L}-1}{v_{I 1}-1}\right) \sum \frac{m !}{b_{1} ! b_{2} ! \ldots b_{m-1} !} v_{I k}\left(\frac{M_{L}}{1 !}\right)^{b_{1}}\left(\frac{h^{\prime \prime}}{2 !}\right)^{b_{2}} \ldots\left(\frac{h^{(m-1)}}{(m-1) !}\right)^{b_{m-1}}
$$

Where the sum is over all the different solutions in nonnegative integers $b_{1}, b_{2} \ldots, b_{\mathrm{m}-1}$ of $b_{1}+$ $2 b_{2}+\cdots+(m-1) b_{m-1}=m$ and $k=b_{1}+\cdots+b_{m-1}$.

Some examples will help to explain how to use equations Eq. 36 \& Eq.40 for the determination of the multiplet equations.

For $\mathrm{m}=1$, we have the initial condition of the iteration $h^{\prime}=M_{L}$; and for $\mathrm{m}=2$, the only solution for $\mathrm{b}, \mathrm{b}_{1}=2, \mathrm{k}=2$, and we get from Eq.40:

$$
h^{(2)}=\left(\frac{M_{L}-1}{v_{I 1}-1}\right) \frac{2 !}{2 !} v_{I 2}\left(\frac{M_{L}}{1 !}\right)^{2}=\left(\frac{M_{L}-1}{v_{I 1}-1}\right) v_{I 2} M_{L}^{2}
$$

And from Eq.37, we have for $\mathrm{m}=1$ :

$$
R_{1}=\varepsilon^{1} F_{S} \frac{1}{1 !}\left(v_{S 1}(1+\alpha) h^{\prime}\right)=\varepsilon F_{S} \frac{M_{L}}{1}\left(v_{S 1}(1+\alpha)\right)
$$

For $\mathrm{m}=2$, we have, $\mathrm{b}_{1}=2, \mathrm{k}=2$, and by means of Eq.43, $\mathrm{R}_{2}$ is immediately obtained: 


$$
\begin{aligned}
R_{2} & =\varepsilon^{2} F_{S} \frac{1}{2 !}\left(v_{S 1}(1+\alpha) h^{(2)}+\frac{2 !}{2 !} v_{S 2}\left(\frac{M_{L}}{1 !}\right)^{2}\right) \\
& =\varepsilon^{2} F_{S} \frac{M_{L}^{2}}{2 !}\left(v_{S 1}(1+\alpha)\left(\frac{M_{L}-1}{v_{I 1}-1}\right) v_{I 2}+v_{S 2}\right)
\end{aligned}
$$

For the case with $\mathrm{m}=3$, the integer, nonnegative solutions of the equation $b_{1}+2 b_{2}=3$, are $\mathrm{b}_{1}=3$ and $\mathrm{b}_{2}=0$ and $\mathrm{k}=3$; second solution $\mathrm{b}_{1}=1$ and $\mathrm{b}_{2}=1$ and $\mathrm{k}=2$, so:

$$
h^{(3)}=\left(\frac{M_{L}-1}{v_{I 1}-1}\right)\left(\frac{3 !}{3 ! 0 !} v_{I 3}\left(\frac{M_{L}}{1 !}\right)^{3}+\frac{3 !}{1 ! 1 !} v_{I 2}\left(\frac{M_{L}}{1 !}\right)^{1}\left(\frac{h^{\prime \prime}}{2 !}\right)^{1}\right)
$$

That simplifies immediately as:

$$
h^{(3)}=\left(\frac{M_{L}-1}{v_{I 1}-1}\right) M_{L}^{3}\left(v_{I 3}+3 v_{I 2}^{2}\left(\frac{M_{L}-1}{v_{I 1}-1}\right)\right)
$$

Equation for multiplet of $m=3$ order is:

$$
R_{3}=\varepsilon^{3} F_{S} \frac{1}{3 !}\left(v_{S 1}(1+\alpha) h^{(3)}+\frac{3 !}{3 ! 0 !} v_{S 3}\left(\frac{M_{L}}{1 !}\right)^{3}+\frac{3 !}{1 ! 1 !} v_{S 2}\left(\frac{M_{L}}{1 !}\right)^{1}\left(\frac{h^{\prime \prime}}{2 !}\right)^{1}\right)
$$

This simplifies immediately to give:

$R_{3}=\varepsilon^{3} F_{S} \frac{M_{L}^{3}}{3 !}\left(v_{S 3}+\left(v_{S 1}(1+\alpha) v_{I 3}+3 v_{S 2} v_{I 2}\right)\left(\frac{M_{L}-1}{v_{I 1}-1}\right)+3 v_{S 1}(1+\alpha) v_{I 2}^{2}\left(\frac{M_{L}-1}{v_{I 1}-1}\right)^{2}\right)$

Following the same path (solving the $b_{1}+2 b_{2}+\cdots+(m-1) b_{m-1}=m$, equation and determining $h$ and $\mathrm{R}_{\mathrm{m}}$ ) the equations for $m$ greater than 3 are easily obtained.

Eq.36 and Eq.40, are also useful to establish and prove (by induction) other general features of a multiplet of order $\mathrm{m}$, but that takes us beyond the aim of this note, the aim of which is to provide the point model expressions relevant to extend passive neutron multiplicity counting practice.

The application of the general formulae developed in this section requires the calculation of the b-coefficients. It is convenient to have a way to calculate these quickly. We offer one approach here. The problem is how to solve equations of the form: $b_{1}+2 b_{2}+\cdots+m b_{m}=m$. The approach is to write the solution as a square matrix (B), where any row corresponds to a value of $\mathrm{k}$, which values range from 1 to $\mathrm{m}$, and the columns are solutions for the b-coefficients. Algebraically this is summarized as follows: 


$$
\begin{aligned}
& \begin{array}{lllll}
b_{1} & b_{2} & b_{3} & \ldots & b_{m}
\end{array} \\
& B=\left[\begin{array}{ccccc}
m & 0 & 0 & \ldots & 0 \\
m-2 & 1 & 0 & \ldots & 0 \\
m-3 & 0 & 1 & \ldots & 0 \\
\vdots & & & & \vdots \\
0 & 0 & 0 & \ldots & 1
\end{array}\right] \begin{array}{c}
k=m-1 \\
k=1
\end{array}
\end{aligned}
$$

and that for Eq.37 and Eq.41, matrix reduce of one order as $\left(\mathrm{B}_{\mathrm{red}}\right)$ :

$$
\begin{aligned}
& \begin{array}{lllll}
b_{1} & b_{2} & b_{3} & \ldots & b_{m-1}
\end{array} \\
& B_{\text {red }}=\left[\begin{array}{ccccc}
m & 0 & 0 & \ldots & 0 \\
m-2 & 1 & 0 & \ldots & 0 \\
m-3 & 0 & 1 & \ldots & 0 \\
\vdots & & & & \vdots \\
0 & 0 & 0 & \ldots & 1
\end{array}\right] \begin{array}{c}
k=m \\
k=2
\end{array}
\end{aligned}
$$

Example for $\mathrm{m}=5, B_{\text {red }}$ is:

$$
B_{\text {red }}(m=5)=\left[\begin{array}{llll}
5 & 0 & 0 & 0 \\
3 & 1 & 0 & 0 \\
2 & 0 & 1 & 0 \\
1 & 0 & 0 & 1
\end{array}\right]
$$

An interesting interpretation/link of the Faa di Bruno equation can be done in terms of multinominal distribution, for an event of multiplicity s, and for the sake of the example to have a system of 3 detectors with efficiency $\varepsilon_{1}, \varepsilon_{2}, \varepsilon_{3}$, and $\overline{\varepsilon_{4}}=1-\varepsilon_{1}-\varepsilon_{2}-\varepsilon_{3}$ the probability of no detection at all, the following relation can then be written:

$1=\left(\varepsilon_{1}+\varepsilon_{2}+\varepsilon_{3}+\overline{\varepsilon_{4}}\right)^{s}=\sum s ! \frac{1}{b_{1} ! b_{2} ! b_{3} ! b_{4} !} \varepsilon_{1}^{b_{1}} \mathcal{E}_{2}^{b_{2}} \varepsilon_{3}^{b_{3}} \overline{\mathcal{E}}_{4}^{b_{4}}$

where $b_{1}+b_{2}+b_{3}+b_{4}=s$, and this is the definition of multi-nominal distribution, and so, $R_{m}$ is seen to be the sum of multi-nominal distributions, but with a constraint that $b_{1}+2 b_{3}+\ldots$ should be valid (the physics interpretation of the equation in our case is to provide the interpretation for neutron correlation counting applications). The full interpretation is also interesting but again such discussion takes us outside the objectives of the present article. 


\section{Conclusion}

Fissioning systems produce neutrons in short duration bursts. The nature of these bursts is dependent both on the mixture of spontaneous fission and $(\alpha, n)$ neutrons sources that are present within the item as well as the nature of the item and its environment. Neutrons which emerge and are detected in an external moderated array of thermal neutron sensors remain correlated in time although they become far more widely distributed in time in a way which is dependent on the materials and design of the detector. The underlying nature of the source terms and the multiplication processes taking place in the item are consequently encoded on the pulse train through the statistical correlations created by the action of the detection process on the emergent neutron chain distribution. Autocorrelation analysis forms the basis important nondestructive assay technique applied to characterization and quantification of special nuclear material. The commonly applied approach make use of point kinetics equations up to $3^{\text {rd }}$ order.

In this work point model equations for quads and pents have been derived using the probability generating function formalism. This extends the analytical options available for neutron multiplicity counting applications, in scenario like dead time corrections. The equations also allow for a straightforward sensitivity analysis, for example gradients such as $\partial P / \partial M_{L}$ can be readily computed, so that the importance of nuclear data selection can be assessed. In addition, a general solution was provided permitting expressions to any order to be derived in a straightforward way.

\section{Future Work}

The higher order multiplet point model equations are expected to correctly capture the important functional dependences but violation of the point model (mathematical) assumptions may still introduce assay bias in geometrically extended (large dense) highly multiplying items. Adaptations to the standard point model that are being explored to correct the assumptions that are built into the standard point model include spatial dependent (known geometry) treatments of the self-multiplication factor.

The general solution presented is useful for analytical benchmarking of criticality calculations. Also it is an interesting mathematical problem of branching processes in its own right and we have used it already in a generalized 'chain derivation-Faa' di Bruno-coincidence detectors fission chain-multiplet' model. The Faa' di Bruno approach allows us to compute $R_{m}$ for all $m$. Now, in principle the number distribution extends to infinity, but for practical problems if we recognize that the multiplicity histograms do have a finite extent, which sets a maximum value of $m$, then using the $R_{m}$ values we obtain from Faa' di Bruno, we can invert the matrix to get the ideal number distribution inclusive of the influence of multiplication. This is an alternative way of generating the number distribution and may have some value in verifying Monte Carlo tools. We have already used this concept in the measurement of non multiplying items (low mass limit) to delineate cosmic ray spallation background. 


\section{Acknowledgement}

We acknowledge the US Department of Energy National Nuclear Security Administration (NNSA) Office of Nonproliferation and International Security for supporting our research. 


\section{References}

[1]. D.M. Cifarelli and W. Hage, Models for three-parameter analysis of neutron signal correlation measurements for fissile material assay, Nucl. Instrum. and Meths. in Phys. Res. A251(1986)550-563.

[2]. W. Hage and D.M. Cifarelli, On the factorial moments of the neutron multiplicity distribution of fission cascades, Nucl. Instrum. and Meths. in Phys. Res. A236(1985)165-177.

[3]. S. Croft, A. Favalli, D.K. Hauck, D. Henzlova, and P.A. Santi, Feynman variance-to-mean in the context of passive neutron coincidence counting, Nucl. Instrum. and Meths. in Phys. Res. $\mathrm{A}(686)(2012) 136-144$.

[4].K. Böhnel, The Effect of Multiplication on the Quantitative determination of Spontaneously fissioining Isotopes by Neutron Correlation Analysis, Nuclear Science and Engineering 90(1985)75-82.

[5]. M.S. Krick, W.H. Geist, and D.R. Mayo, A weighted point model for the thermal neutron multiplicity assay of high-mass plutonium samples, Los Alamos National Laboratory report LA14157(Oct. 2005).

[6]. W. Hage and D.M. Cifarelli, Correlation analysis with neutron count distribution for paralyzing dead-tine counter for the assay of spontaneous fissioning material, Nuclear Science and Engineering 112(1992) 136-158.

[7].P. Baeten, M. Bruggeman, and R.Cachon, Single- and multi-deadtime parameter corrections of one-and two-dimensional Rossi-alpha distributions for time interval analysis in neutron coincidence counting, Nucl. Instrum. and Meths. in Phys. Res. A 390 (1997) 345-358.

[8]. D.K. Hauck, S. Croft, L.G. Evans, A. Favalli, P.A. Santi, and J. Dowel, Study of a theoretical model for the measured gate moments resulting from correlated detection events and an extending dead time, Nucl. Instrum. and Meths. in Phys. Res. A 719(2012) 57-69.

[9]. S.Croft, A.Favalli, Dead time corrections for neutron multiplicity counting, Nucl. Instrum. and Meths. in Phys. Res. A 686 (2012) 115-116.

[10]. S.Croft, A.Favalli, Higher Order Dead Time Corrections for Neutron Multiplicity Counting, ESARDA bulletin 47, 2012.

[11]. S.Croft, R.D.McElroy, S.C.Kane, Coincidence Gate Utilization Factors for neutron correlation counters with up to three components in the die-away profile, Proceeding of the ICEM'07: The 11 ${ }^{\text {th }}$ International Conference on Environmental Remediation and Radioactive Waste Management, Bruges ( Brugge), Belgium, September 2-6, 2007. 
[12]. S.Croft, D.Henzlova, Determining ${ }^{252} C f$ source strength by absolute passive neutron correlation counting, Nucl. Instrum. and Meths. in Phys Res. A714 (2013)5-12.

[13]. W.P.Johnson,“The Curiosity History of Faa' di Bruno's Formula " Amer. Math. Monthly 109, 217-234, 2002; available at

https://www.maa.org/sites/default/files/pdf/upload_library/22/Ford/Johnson217-234.pdf (last accessed March 24, 2015).

[14]. Cav.F.Faà di Bruno, "Sullo sviluppo delle funzioni." Ann. di Scienze Matem. et Fisiche (Edited by B.Tortolini) 6, 479-480, Roma 1855. 